\title{
Compromiso Escolar: Trayectoria y Política Educativa en Iberoamérica
}

\author{
School Engagement: Trajectory and Educational Policy in Iberoamerica
}

\author{
Mónica Bravo-Sanzana ${ }^{1}$, Mahia Saracostti ${ }^{2}$, Laura Lara ${ }^{3}$, Rosa María Diaz-Jiménez ${ }^{4}$, \\ Jhonatan S. Navarro-Loli ${ }^{5}$, Fernando Acevedo ${ }^{6}$ y José Aparicio ${ }^{7}$
}

\begin{abstract}
Resumen
Las trayectorias educativas se ven afectadas por múltiples factores, incluyendo aspectos estructurales, de contexto e individuales. En este artículo, se realiza una revisión del panorama educativo de cinco países iberoamericanos (Chile, Colombia, España, Perú y Uruguay), desde la perspectiva de políticas públicas en educación, deserción, abandono, fracaso escolar y desempeño académico, para posteriormente vincular con el análisis conceptual del compromiso escolar. De este modo, se invita a comprender el compromiso escolar como un predictor significativo en la identificación de variables que intervienen en el éxito de las trayectorias educativas y como una estrategia de monitoreo del proceso de aprendizaje, para contribuir con trayectorias educativas diversas, concebidas desde la singularidad, la inclusión y conducentes al bienestar integral de la persona.
\end{abstract}

Palabras clave: política educativa, abandono escolar, fracaso escolar, logro académico, compromiso escolar

\begin{abstract}
Educational trajectories are affected by multiple factors, including structural, contextual and individual aspects. In this article, a review of the educational panorama of five Ibero-American countries (Chile, Colombia, Spain, Peru and Uruguay) is made from the perspective of public policies in education, dropout, school failure and academic performance, to later link these with the conceptual analysis of school engagement. Therefore, this article is an invitation to understanding school engagement as a significant predictor in the identification of variables that intervene in the success of educational trajectories; as well as a strategy for monitoring the learning process, to contribute with diverse educational trajectories, conceived from singularity, inclusion and conducive to the integral well-being of the person.
\end{abstract}

Keywords: educational policy, school dropout, school failure, academic achievement, school engagement

Financiamiento: Este artículo ha recibido el apoyo de los proyectos FONDEF ID14I20078 y FONDEF IT 19I0012 financiados por la Comisión Nacional de Investigación Científica y Tecnológica CONICYT, Chile.

\footnotetext{
${ }^{1}$ Doctora en Ciencias de la Educación. Investigadora y Docente. Núcleo Científico Tecnológico en Ciencias Sociales y Humanidades. Universidad de La Frontera. Avenida Francisco Salazar 01145, Temuco, Chile. Tel.: +56 991041288. Correo: monicaviviana.bravo@ufrontera.cl ${ }^{2}$ Doctora en Social Welfare. Profesora Titular de la Escuela de Trabajo Social. Facultad de Ciencias Sociales. Universidad de Valparaíso. Blanco 951, Valparaíso, Chile. Tel.: +56 998293161. Correo: mahia.saracostti@uv.cl

${ }^{3}$ Laura Lara. Doctora en Psicología. Profesora Titular. Universidad Autónoma de Chile, 5 poniente 1670, Talca, Chile. Tel.: +56 712735770. Correo: 1larav@uautonoma.cl

${ }^{4}$ Doctora en Ciencias Sociales. Profesora Titular de Trabajo Social y Servicios Sociales. Facultad de Ciencias Sociales, Universidad Pablo de Olavide de Sevilla, Ctra. de Utrera 1, CP. 41013 Sevilla, España. Tel.: +34 610125899. Correo: rdiajim@upo.es

${ }^{5}$ Magíster en Psicología. Docente. Instituto de Investigación de Psicología. Universidad de San Martín de Porres. Av. Tomás Marsano 242, Lima 34, Perú. Tel.: +51 5136300. Correo: jnavarrol@usmp.pe

${ }^{6}$ Doctor en Educación. Profesor Adjunto. Universidad de la República de Uruguay. Centro Universitario de Rivera, Ituzaingó 667, C.P. 40.000, Rivera, Uruguay. Tel.: +59899866115. Correo: face@ cur.edu.uy

${ }^{7}$ Doctor en Psicología. Decano del Instituto de Estudios en Educación. Universidad del Norte. Km.5 Vía Puerto Colombia, Barranquilla, Colombia. Tel.: +57 53509799. Correo: aparicio@uninorte.edu.co
} 


\section{Introducción}

Desde 1948, la educación es considerada un derecho universal (ONU, 1948), lo que ha convocado a los Estados a gestionar esfuerzos a través de políticas educativas para garantizar este derecho y posibilitar a cada uno de sus ciudadanos, su adecuada inserción social.

En el presente artículo se realiza una revisión del sistema educativo de cinco países iberoamericanos: Chile, Colombia, España, Perú y Uruguay, para describir sus políticas públicas y principales indicadores asociados a la promoción de trayectorias de aprendizaje de calidad en el contexto escolar, y vincular con el análisis conceptual del compromiso escolar.

\section{Trayectorias escolares}

Desde un punto de vista teórico, la trayectoria escolar o educativa parece haber tenido su origen en la sociología de la educación en los años 70 (Bourdieu \& Passeron, 1979), para dar cuenta de las desigualdades sociales con las que se accede a la escuela, dependiendo del capital cultural de las familias de origen. Estos autores hicieron por primera vez referencia a la distancia que puede existir entre la expectativa de transito normal que tienen los sistemas educativos (basada en edades de ingreso y niveles educativos obligatorios), y los caminos reales que siguen muchos niños y niñas, los cuales, en buena medida, se definen por los capitales culturales heredados.

A partir de esta idea, Terigi (2014) propuso dos tipos de trayectorias escolares: las teóricas y las reales. Las primeras, comprenden aquellos recorridos de progresión lineal que el sistema asigna, con espacios temporales y una periodización estándar. Según estas, se espera que los estudiantes ingresen al sistema escolar con una edad establecida por las normas, transiten por los diferentes niveles escolares de manera continua, sin repetir curso, ni abandonar la escuela, y finalicen los niveles educativos a la edad que corresponde. Las segundas, son los recorridos reales que realizan los estudiantes, incluyendo todos los posibles problemas escolares asociados, tales como falta de compromiso escolar, repitencia, ausentismo, sobreedad, necesidades educativas especiales, abandono escolar y bajo rendimiento.
En la literatura se hace referencia a las trayectorias educativas, con diversos adjetivos. Se habla de trayectorias escolares completas o exitosas (Cárdenas, 1998); pero también de trayectorias escolares no encauzadas (Terigi, 2009). En cuanto a las primeras, la referencia a trayectorias escolares completas aparece vinculada comúnmente con iniciativas centradas en evitar la deserción de los niños, por lo cual, pareciera que la preocupación desde esta perspectiva no estaría centrada tanto en la calidad del proceso, sino en la permanencia de los alumnos en el sistema. Por su parte el concepto de trayectorias educativas exitosas, tampoco ha estado exento de críticas, pues desde ciertas conceptualizaciones, el éxito sólo se vincula a la meta de que los niños y jóvenes completen sus estudios sin problemas de repitencia, extra edad y deserción.

Nuestra propuesta, siguiendo a Toscano, Briscioli y Morrone (2015) es que la trayectoria escolar debe comprenderse como el estudio de los aspectos singulares de la historia de vida de cada individuo, entrelazados con las experiencias de aprendizaje y desarrollo que las instituciones educativas deben ofrecer; y por tanto, el éxito, más que ser concebido como el logro de estándares y metas académicas homogeneizadas, debe ser entendido como el proceso de construcción individual de un proyecto vital de sentido, como persona y ciudadano.

Trayectoria educativa en los documentos de política pública de cinco países iberoamericanos

En el caso de Chile, Espinoza (2015) y Espinoza, González, McGinn y Castillo (2019) concluye que, en los últimos siete gobiernos democráticos, al alero de una agenda neoliberal, no se han generado políticas educativas desde 1981, más bien se han centrado en establecer diferentes programas y acciones caracterizados por no presentar conexión entre sí y que no han sido evaluados, por lo que se desconoce si los recursos invertidos generaron algún impacto.

A comienzos de la década del 2000 en Chile, comenzó a desarrollarse un fuerte descontento social, por lo que su política educativa se orientó a enfrentar lo desigual e hiper segregado de su sistema educativo (Murillo \& Garrido, 2017). Entre las acciones llevadas a cabo, incorporó en la 
gestión de los recursos públicos el Subsidio Preferencial Escolar, SEP, cuya finalidad es compensar las desigualdades sociales de origen existentes (Ley $\mathrm{N}^{\circ}$ 20.248, 2008). No obstante, se evidencia la dificultad de cumplir estos objetivos y su implementación parcial (De la Vega Rodríguez \& Picazo Verdejo, 2016). Posteriormente, se hizo más crítica la mirada de la ciudadanía sobre el "rol, sentido y efectos" de la educación (Weinstein \& Villalobos, 2016). En este contexto, se aprueba la Ley 20.370 General de Educación de 2009 con el objetivo de tener un sistema educativo caracterizado por la equidad y calidad de su servicio, y se avanzó, además, hacia la Ley $\mathrm{N}^{\circ} 20.536$ sobre violencia escolar, promulgada el año 2011 para promover la convivencia; y la Ley de Inclusión, Ley 20.845 de 2016, para dar solución al problema respecto de la distribución de estudiantes vulnerables, aunque el sistema persiste debido a que es muy similar a la del sistema antiguo (Sillard, Garay, \& Troncoso, 2018)

Otra política compensatoria del sistema educacional en Chile, es el sistema de becas a través de la Junta de Auxilio Escolar y Becas (JUNAEB). Resulta de particular interés la Beca de Apoyo a la Retención Escolar (BARE); y la provisión a cada estudiante de instituciones de gestión pública, de útiles escolares al inicio del año lectivo (García, 2018).

Colombia por su parte, parece caracterizarse por tener un decreto o resolución de política pública para cada una de las variables implicadas en el sistema educativo. Por ejemplo, hay normativas que promueven, la enseñanza de una segunda lengua (Bonilla \& Tejada, 2016), el acceso y manejo de las tecnologías de la Información y la comunicación (Caro \& Flores, 2018), la formación docente (Casarrubia-Ruíz \& Ramos, 2018), la educación rural (Naranjo \& Carrero, 2017) o la educación inclusiva (Ortiz \& Betancourt, 2020). Desafortunadamente, esta prolijidad jurídica, parece carecer de una política articuladora, centrada en la promoción de trayectorias educativas de calidad para cada estudiante. Desde que se promulgo la ley 115 de 1994, que sirve de marco general para la educación en este país, las políticas educativas no parecen haber logrado materializar en la práctica, un modelo de promoción y seguimiento de los itinerarios de desarrollo y aprendizaje, para cada aprendiz.

Tal vez, debido a las grandes desigualdades sociales que aún subsisten en este país, buena parte de los recursos y programas se han concentrado en asegurar el acceso y evitar la deserción escolar, a través de intervenir variables socio-económicas. Para garantizar la permanencia en el sistema educativo de los niños más pobres, se han promulgado leyes, como la 715 del 2001, para financiamiento del transporte escolar, o el decreto 4807 de 2011, que reglamentó la gratuidad educativa en la educación básica (Castelar, 2015).

Por las mismas razones, y como un mecanismo para evitar la alta deserción en el tránsito de la educación básica a la media, se creó la ley 1014 de 2006, para promover el espíritu emprendedor entre los estudiantes. (Guarnizo, Velásquez, Jiménez, \& Alzate, 2019).

Por otra parte, se han desarrollado iniciativas de orden nacional, como el programa de alimentación escolar (PAE) y el programa Familias en Acción que provee subsidios condicionados a la asistencia escolar (Pérez, 2018). Desafortunadamente, estas estrategias no parecen ser suficientes para llevar a Colombia a ser la nación "más educada" para el año 2025 (Guevara \& Téllez, 2019).

Sólo en los documentos de política pública más recientes, la preocupación por la deserción y la permanencia, aparece acompañada de la referencia a la necesidad de garantizar trayectorias educativas completas (Ministerio de Educación Nacional de Colombia, 2019).

Revisando el caso de España, según GimenezMartínez (2015), el desarrollo de su sistema educativo ha estado marcado desde sus orígenes por la lucha contra el analfabetismo y por garantizar la escolarización universal, en un marco de políticas neoliberales que socavan el derecho a la educación para todos. En la década de 1980, el sistema enfrenta un problema público: el "fracaso escolar", el cual es asociado a la no titulación en la educación obligatoria. Entre las diversas políticas desarrolladas, se encuentran las denominadas "medidas de atención a la diversidad" (MAD). Y al alero de la Ley Orgánica General del Sistema Educativo (LOGSE), en los noventa, se encuentran los programas de 
diversificación curricular (PDC) y los programas de garantía social (PGS). Los programas compensatorios estuvieron comprendidos por un sistema de becas y recursos para estudiantes con dificultades de primeros años de secundaria, con currículo adaptado para aquellos con "desfase curricular" y en "desventaja" por factores socioeconómicos y "culturales" (MartínezNovillo, 2017a).

Otra de las principales problemáticas del sistema educativo español dice relación con la población de inmigrantes que llegan al país como niños, y que representan un desafío al sistema (Essomba, Guardiola, \& Pozos, 2019). Estudios dan cuenta del riesgo de las trayectorias educativas de esta población (De Miguel-Luken \& Solana-Solana, 2016). En este contexto, Pàmies y Bertran, (2018) señalan cuatro tendencias en la investigación para trayectorias de éxito en inmigrantes, destacando la experiencia escolar positiva; y, Essomba et al. (2019), muestran entre los principales factores a considerar, la capacitación de docentes para desarrollar un enfoque intercultural.

Así, las políticas públicas de apoyo educativo, pueden ser una oportunidad, o, por el contrario, una interpelación del mismo rendimiento escolar (Monarca, 2017). Se hace necesario, entonces, de un análisis que supere las concepciones positivistas de las políticas y reformas educativas e indaguen sobre su origen, lógica y naturaleza (Tarabini, Curran, Montes, \& Parcerisa, 2016).

En el caso peruano, en la actualidad la trayectoria educativa se asegura en la Ley General de Educación, Ley $N^{\circ} 28044$ aprobada en 2003 en que se indica que la educación peruana se divide en etapas (inicial, primaria y secundaria) y promueve el desarrollo integral del estudiante a través de un enfoque obligatorio, gratuito $\mathrm{e}$ inclusivo. En este último aspecto, el enfoque inclusivo es importante en el Perú debido a la diversidad cultural, no obstante, a pesar de esta normativa, hay evidencia de que el Estado y Gobierno Regional, no cumplen con las condiciones establecidas en dicha ley cuando se trata de educación rural y población indígena debido al aislamiento de estas personas, su distanciamiento con la tecnología y falta de infraestructura física y virtual necesarias para el proceso de enseñanza aprendizaje (Charry, 2019) y que la educación tradicional puede ser contraria a la cosmovisión de dichas comunidades (Bermejo-Paredes \& Maquera-Maquera, 2019).

En la producción académica de Uruguay la noción de trayectorias educativas es también reciente. En diciembre de 2015 el Consejo Directivo Central de la Administración Nacional de Educación Pública resolvió la creación del Sistema de Protección de Trayectorias Educativas (SPTE), donde el énfasis estuvo puesto en el trayecto de la educación media (tanto educación secundaria general como educación técnica), incluyendo los tránsitos interciclos, ya que es allí donde las tasas de rezago y de abandono de los estudios son más preocupantes. El objetivo tácito del SPTE -"la universalización de la educación media básica y la generalización del egreso de la educación media superior"- aún está lejos de alcanzarse, y "encuentra su principal cuello de botella en el desgranamiento educativo durante la educación media" (De Armas, 2017, p. 26).

La obligatoriedad en educación. Actualmente, el tramo de escolarización obligatoria tiene una duración de 14 años en Perú y Uruguay; 13 años en Chile; 10 años en Colombia. Al mediar la década de 2010, en Chile, el 84,3\% de los jóvenes de 25 a 35 años la completaron. En Colombia y Perú, entre el $71 \%$ y el $59 \%$. Mientras que Uruguay, entre el $37,9 \%$ y el $24,3 \%$ de los jóvenes de 25 a 35 años finalizó el tramo de escolarización obligatoria (SITEAL, 2019). Al respecto, se destaca que el Estado uruguayo ha implementado un vasto repertorio de políticas y programas educativos que, en su conjunto, propenden a la universalización de los tres primeros ciclos obligatorios y a la generalización del último, como paso previo a su universalización (Acevedo \& Menni, 2016).

En España, la instauración de la educación obligatoria en la Ley 14/1970, General de Educación y Financiamiento de la Reforma Educativa (LGE), ofreció una formación única de 6 a 14 años para todos los estudiantes, lo que supuso una política favorable para frenar la segregación temprana (Puelles, 2011b, citado en Morentin-Encina, Ballesteros, \& Velázquez, 2019). Posteriormente la escuela obligatoria se amplió de los 6 a los 16 con la Ley Orgánica de Ordenación General del Sistema Educativo, LOGSE, en 1991, manteniéndose hasta hoy. No 
obstante, surgieron reveses no considerados pues los estudiantes que no consiguen certificarse en la secundaria obligatoria tienen problemas para proseguir estudios reglados post-obligatorios (Morentin-Encina, Ballesteros, \& Velázquez, 2019), siendo el porcentaje de estudiantes que no logran el diploma de educación obligatoria alrededor del 20\% (Fernández-Mellizo \& Martínez-García, 2017).

\section{Fracaso escolar y sus extensiones como la deserción, repetición o el abandono escolar}

De acuerdo a Román (2013), “...bajos aprendizajes, reprobación de grados, ausencias reiteradas, desmotivación por avanzar y aprender, todos signos evidentes de un proceso de fracaso escolar, cuyo último eslabón es el abandono y la desvinculación definitiva de la escuela (deserción) y el sistema" (p. 37). En este mismo contexto la autora señala que los estudios identifican factores exógenos del sistema escolar (estructura social, económica y política), y factores endógenos, esto es, inherentes a la escuela (fracaso escolar construido desde la escuela).

La deserción escolar hace referencia a un año en particular, no implicando un abandono definitivo del sistema escolar, no obstante, compromete severamente la trayectoria escolar. En este sentido, D'alessandre (2014) pone de relieve que los estudiantes de enseñanza secundaria en Latinoamérica cuestionan fuertemente la función socializadora de la escuela, consideran que el ambiente escolar es violento, que los profesores no saben enseñar ni motivar, que lo que aprenden no es útil para la vida y que la educación que se ofrece en sus países de residencia es cuestionable.

La repetición ha sido concebida por los sistemas escolares como un dispositivo remedial, y puede ser comprendido como una consecuencia del fracaso académico, la sobreedad, inasistencia a la escuela o la falta de oportunidades educativas locales (UNESCO, 2012). Sin embargo, tiene una asociación negativa tanto con el logro de los aprendizajes como su ineficacia en contribuir al mejoramiento del rezago escolar (Terigi, 2014).

A pesar de las discrepancias señaladas de acuerdo a Flotts et al., (2016) con datos TERCE 2013, Chile muestra una cifra inferior al $15 \%$. No obstante, de acuerdo a los resultados de PISA 2012, entre un $20 \%$ y $29 \%$ de los estudiantes de
15 años en Chile y Perú repitieron curso al menos una vez; entre un 30\% y $39 \%$ de estudiantes en Uruguay; y en Colombia, más del $40 \%$ de los estudiantes repitieron curso al menos una vez.

En esta perspectiva, en Chile, diferentes estudios muestran: efectos positivos a largo plazo de programa público nacional de atención y educación de la primera infancia para la reducción de la brecha socioeconómica del logro (Cortazar, Molina, Sélman, \& Manosalva, 2020); sugerencias de diseño de apoyos psicosociales de programas para prevenir el absentismo y el abandono escolar, considerando las voces de los estudiantes y la necesidad de preparar profesionales para un trabajo interprofesional coordinado (Montecinos, Castro, Díaz, Manríquez, \& Edwards, 2018); y, también, que los estudiantes que reciben apoyo financiero tienen mayores probabilidades de completar sus estudios de manera oportuna (Venegas-Muggli, 2020).

Cuando se revisan estudios en Chile en contextos de estudiantes fuera de la escuela, destacan dos investigaciones realizadas al alero del sistema de Centros de Segunda Oportunidad, sugieren investigación futura sobre las actividades más efectivas que realizan los centros para involucrar a diferentes estudiantes, debido a que la retención en la escuela depende mucho de lo que se experimente en ella (Espinoza, González, McGinn, \& Castillo, 2020).

Respecto a España, es el país de la OCDE con la mayor proporción de repetidores en la escuela secundaria (11\%) y cinco veces la proporción promedio de repetidores (2\%) de los países de la OCDE, considerando el año académico 2016-17 (Cabrera, 2019).

Martínez-Novillo (2017b) analiza el fracaso escolar es España como un problema social que surge en la década de 1960 y cristaliza como un problema público en 1980, llegando a tener la tasa de abandono escolar más alta de la Unión Europea. A los determinantes socioeconómicos, se relevan actualmente el mercado laboral, la nacionalidad y el nivel de educación de la madre (Bayon-Calvo, 2019).

Entre las estrategias para frenar el abandono se proponen realizar acciones de política educativa inclusivas: promover la lectura a una edad temprana, especialmente en los niños 
(quienes repiten más que las niñas), apoyar a los escolares nacidos en los últimos cuatro meses del año calendario (Cabrera, 2019); y analizar el abandono escolar prematuro desde la experiencia escolar, recogiendo la perspectiva de los jóvenes a través de sus narrativas (Morentin-Encina, Ballesteros, \& Mateus, 2019).

En Perú, estudios indican que la deserción académica afecta alrededor de 5\% de estudiantes de Educación Básica Primaria, en igual proporción para varones y mujeres, pero el $83.3 \%$ son de áreas rurales y solo el $16.7 \%$ de zonas urbanas, siendo una de las principales causas el trabajo infantil (Tovar \& Ríos, 2017). Otro estudio indicó que la tasa media de fracaso escolar de acuerdo a las regiones fue de $41.3 \%$ para la selva, $32.1 \%$ para la sierra y de $28.5 \%$ para la costa, y que el factor sociocultural es un predictor importante de dicho fracaso (Latorre, 2018).

Por su parte en Uruguay, a pesar de que el acceso a la Educación Media está cerca de ser universal, el abandono de los estudios en este nivel es uno de los principales problemas (Acevedo, 2015; 2020), y cuya magnitud queda en evidencia si se comparan las tasas de acreditación de la Educación Media en Uruguay con las de la mayoría de los países latinoamericanos (De Melo \& Machado, 2016). Aproximadamente uno de cada tres estudiantes que acceden a la Educación Media no completa el primer tramo -Ciclo Básico-de tres años (De Armas, 2017).

Entre las políticas asociadas a evitar el fracaso escolar en Uruguay, merecen especial destaque el Programa de Maestros Comunitarios (enfocado en Educación Primaria) y, en Educación Media, el Programa de Impulso a la Universalización del Ciclo Básico (focalizado en la prevención del abandono y la repetición de la población más vulnerable de ese nivel), Compromiso Educativo (orientado a la persistencia en Educación Media Superior), Tránsito Educativo (focalizado en la transición entre Educación Primaria y Educación Media), Aulas Comunitarias (en procura de la reinserción a Educación Media de estudiantes de 12 a 15 años que abandonaron sus estudios o que presentan riesgos de hacerlo), el Programa de Acompañamiento Pedagógico a la Trayectoria Estudiantil, Liceos con Tutorías y, en Educación Media Técnico-Profesional, la Formación Profesional Básica (Acevedo \& Menni, 2016).

\section{Desempeño académico y factores asociados}

Los contextos sociales condicionan en gran medida los resultados de aprendizaje -y, por ende, la reproducción intergeneracional de las desigualdades sociales-, a su vez, los factores educativos se constituyen como factores importantes (Rivas \& Sanchez, 2020). A este respecto, la literatura señala que los procesos escolares (clima escolar, gestión del director, satisfacción docente y desempeño docente) son el elemento que explica el mayor porcentaje de variación en el aprendizaje, salud y bienestar de los adolescentes (Virtanen et al., 2009; Benavente et al., 2017).

En cuanto a los desempeños académicos, los resultados basados en el Segundo Estudio Regional Comparativo y Explicativo (SERCE) implementado en 2006 y en el estudio TERCE del año 2013, para tercer y sexto grado, evidenciaron que Chile se encuentra consistentemente por encima del promedio regional, en todas las pruebas y grados evaluados. También Uruguay se encuentra entre los países que en la mayoría de los casos se encuentran en este mismo grupo, salvo en la prueba de escritura en sexto grado (igual al promedio). No obstante, ambos países presentan una alta variabilidad de puntajes al interior del país. Esto significa que los aprendizajes se distribuyen de manera muy desigual entre los estudiantes, sugiriendo que sus sistemas educacionales presentan gran desigualdad en las oportunidades de aprendizaje. Continuando, Perú se encuentra por sobre la media regional en las pruebas de escritura, lectura y matemática en tercer grado y matemática en sexto grado, en los demás dominios puntúa igual al promedio regional. La situación de Colombia es diferente, sus puntuaciones se distribuyen en todos los grupos, destaca por sobre el promedio en la prueba de lectura y ciencias en sexto grado, no obstante, para el mismo grado en la prueba de escritura puntúa bajo el promedio. En las demás pruebas se encuentra igual al promedio regional (Flotts et al., 2016).

Al analizar con datos de una evaluación nacional de lectura y matemática en Chile, año 2019 para octavo grado medidos por el sistema de medición de la calidad de la educación (SIMCE), observamos en resultados desagregados por grupos socioeconómicos (bajo, medio bajo, 
medio, medio alto y alto), brechas de 46 y 53 puntos, tanto en hombres y mujeres, entre los grupos bajo, medio bajo y el grupo alto; en la prueba de Matemática las brechas son mayores, de 63 y 74 puntos, en ambos sexos, para los mismos grupos (Agencia de Calidad de la Educación de Chile, 2019a).

Sumado a las evaluaciones nacionales, desde la década de 1990, Chile ha participado en todos los principales estudios internacionales de evaluación a gran escala. Esta participación realizada con gobiernos de diferentes ideologías, tienen un profundo impacto en las políticas educativas. Los resultados se han utilizado para adquirir conocimiento y perspectiva, por ejemplo, elaborar estándares de aprendizaje nacionales, planes de estudio, mapas de progreso, etc.; no obstante, uno de los principales desafíos que se enfrenta es la creación de capacidad docente (Cox $\&$ Meckes, 2016).

En este contexto, Chile participó en la prueba internacional PISA (2018), del Programa Internacional para la Evaluación de Estudiantes de la OCDE, y entre algunos de sus resultados se encuentran: obtuvo un promedio de 452 puntos, resultado bajo el promedio de la OCDE (487), pero se posiciona por sobre todos los países de Latinoamérica participantes. Uno de los principales factores asociados a los resultados de lectura es el nivel socioeconómico y cultural (Agencia de Calidad de la Educación de Chile, 2019b).

Desde el punto de vista de los estudios empíricos con datos de Chile, la literatura nos revela varios indicios a considerar para propiciar ambientes que favorezcan las experiencias de los estudiantes. Entre estos estudios se destacan asociaciones de variables socioemocionales individuales y asociaciones relacionadas con la escuela y con el rendimiento escolar (López et al., 2017); recomendaciones de mejora del entorno escolar general, considerado como un mejor predictor de los resultados de pruebas (Gazmuri, Manzi \& Paredes, 2015); la promoción de las expectativas educativas de los padres (BravoSanzana, Salvo-Garrido, \& Muñoz-Poblete, 2015; Bravo-Sanzana, Salvo-Garrido, Mieres-Chacaltana, Mansilla, \& Hederich, 2017); apoyo a políticas anti-bullying y a la promoción de la identidad estudiantil (Contreras, Elacqua, Martinez, \&
Miranda, 2016); y a relevar la atención en la gestión del clima social escolar (Bravo-Sanzana, Pavez, Salvo-Garrido, \& Mieres-Chacaltana, 2019), siendo necesario, entre otros, contar con instrumentos para medir e identificar comportamientos disruptivos en la escuela (Gálvez-Nieto, Vera-Bachman, Trizano, \& García, 2015).

Por último, siempre en términos de equidad, estudios en Chile desde la perspectiva de género señalan que ésta sigue marginada de la toma de decisiones de las políticas educativas, evidenciándose la persistencia de brechas de aprendizaje en las trayectorias educativas de hombres y mujeres; y su efecto negativo para las mujeres en el mercado laboral (Baeza \& Lamadrid, 2018).

En el caso de España, al igual que otros países occidentales, ha desarrollado sus políticas educativas en el marco de las grandes organizaciones internacionales y supranacionales (López \& González, 2018). Una de estas evaluaciones es el Programa para la Evaluación Internacional de Estudiantes (PISA). Participan estudiantes de 15 años. Las áreas son lectura, matemáticas y ciencias, junto a otros indicadores como bienestar. Los resultados claves mostraron que los estudiantes españoles puntuaron por debajo de la media de la OCDE en matemáticas (481 puntos) y ciencias (483 puntos). El nivel socio-económico de los y las estudiantes explica el $12 \%$ de la variación de rendimiento en matemáticas y el $10 \%$ de la variación de rendimiento en ciencias comparado, respectivamente, con el $14 \%$ y el $13 \%$ de variación de media entre los países de la OCDE. Entre otros resultados, informaron que el clima disciplinario en las clases de lengua estaba lejos de lo ideal (OECD, 2018).

Respecto a estudios empíricos asociados a rendimiento académico en España, la revisión muestra datos de gran interés: las condiciones del mercado laboral y, al mismo tiempo, el desempeño previo determinan las decisiones educativas futuras (Guio, Choi, \& Escardíbul, 2018); las diferencias nacionales y regionales en el abandono escolar prematuro pueden ser la consecuencia de diferencias subyacentes en la estructura económica y social de una región, y no en el sistema educativo (Bayón-Calvo, Corrales- 
Herrero, \& De Witte, 2020); otro estudio muestra que el entorno familiar, especialmente la formación de los padres más que su nivel de renta, sigue ejerciendo una significativa influencia sobre el rendimiento académico (Ferreiro, Ríos, \& Álvarez, 2016); que la desigualdad por clase social de origen es máxima entre los peores estudiantes (Simões, Rivera, Moreno, \& Matos, 2018); los estudiantes con antecedentes migratorios obtienen resultados escolares más pobres que sus pares nativos, y su promoción educativa entre etapas es más baja que el promedio (Essomba et al., 2019).

En Perú, para la evaluación del desempeño de los estudiantes, Muelle (2018) indica que las mujeres tienen mayores puntajes que los varones en cuanto a lectura, pero un $62 \%$ más de probabilidad en comparación a los varones de obtener un rendimiento deficiente en materias de ciencia y matemáticas. También se ha reportado que el factor económico es importante para el éxito en pruebas de matemática y lenguaje porque este se relaciona con la ubicación geográfica de la familia y por ende la elección de la escuela está restringida por este factor (León \& Valdivia, 2015), además, la educación de los padres y el estado de salud del estudiante también son factores importantes pero su impacto se ve disminuido cuando se trata de estudiantes de comunidades indígenas (Arteaga \& Glewwe, 2019).

\section{El compromiso escolar como un predictor significativo para contribuir con trayectorias educativas}

La evidencia mostrada indica que es preciso focalizar los esfuerzos en variables que los profesores y la escuela puedan trabajar directamente con los estudiantes y sus familias, y ofrecer, de esta manera, oportunidades integrales más cercanas, contextualizadas y vinculantes para promover los logros de aprendizajes y trayectorias educativas completas.

Desde esta perspectiva, el compromiso escolar ha sido evidenciado como una variable clave para contribuir al mejoramiento de la calidad de la educación (Saracostti, Lara, \& Miranda-Zapata, 2019; Lara et al., 2018; Miranda-Zapata, Lara, Navarro, Saracostti, \& De Toro, 2018), especialmente en la prevención de la desescolarización, ya que la decisión de abandonar la escuela es la etapa final de un proceso de pérdida paulatina de la vinculación con el interés por los estudios.

Es concebido como un fenómeno biopsicosocial (Rumberger \& Rotermund, 2012), multidimensional, conformado por distintas pero integradas dimensiones que interactúan entre sí, incluyendo componentes afectivos, conductuales y cognitivos (Janosz, Archambault, Morizot, \& Pagani, 2008). Se sugiere como un concepto primordial, tanto para comprender el fenómeno de la desescolarización como para promover trayectorias educativas que permitan el desarrollo de los estudiantes. En este contexto, disponer en los centros educativos de un dispositivo de evaluación del compromiso escolar, en sus tres dimensiones, para contar con información válida y confiable que permita el levantamiento de estrategias contextuales a nivel local, contribuye con la prevención del fracaso escolar y sus extensiones y con la protección de las trayectorias educativas (Saracostti et al., 2019).

\section{Conclusiones}

La referencia en política pública sobre la conceptualización de trayectorias educativas en los países analizados presenta una data reciente, las últimas dos décadas, salvo España que inicia de manera más temprana en la década de 1960. En todos los países la política pública de trayectorias educativas se ha orientado más a evitar la deserción (y a mejorar los indicadores asociados), que, a pensar y comprender la trayectoria educativa desde un punto de vista individual y único, en el cual subyace una experiencia de vida, un desarrollo personal, una experiencia subjetiva.

La política educativa de los países analizados reporta problemas internos propios de sus contextos que afectan sus propósitos como, por ejemplo, conflicto armado (como el caso colombiano), población escolar inmigrante (como el caso de España), crítica social e ideológica a sistemas de evaluación nacional e internacional y a la segregación social producida por el sistema económico (Chile); no obstante, todos presentan una problemática común, esto es, la inequidad de sus sistemas educativos, donde el fracaso y abandono educativo está determinado 
principalmente por el origen socioeconómico. Entonces, a pesar de los esfuerzos realizados en los países iberoamericanos que hemos estudiado, persiste en cada uno de ellos, aunque en diferente magnitud, sociedades marcadas por la desigualdad en la cual el Derecho a la Educación, continúa siendo un desafío. Temas asociados a esta problemática, y pendientes, serían las políticas de género y de inclusión, para evitar todo tipo de discriminación en el sistema escolar.

También se evidenció que las acciones de los países para evitar el fracaso escolar y sus extensiones son diversas. Políticas compensatorias que van desde programas de garantía social, curriculum adaptados, apoyo con recursos financieros a las escuelas, acompañamiento pedagógico, etc. No obstante, lo anterior, todavía es necesario una mayor inversión que posibilite las condiciones óptimas para que todos y todas puedan recorrer trayectorias educativas plenas, esto es, que posibiliten a las personas desarrollar proyectos de vida sustentables y con bienestar. Se hace necesario mirar la trayectoria educativa más allá de la asistencia escolar, el rendimiento escolar y la promoción entre niveles y ciclos educativos.

En esta perspectiva, es absolutamente necesario mejorar las experiencias que tienen los estudiantes en los centros educativos. El gran desafío para los centros es llegar a convertirse en espacios relevantes, seguros, con climas sociales que promuevan la convivencia y el aprendizaje para todos los integrantes de la comunidad educativa. En este sentido, la trayectoria educativa debe ser un acto gestionable, planificado desde la protección de las experiencias socioemocionales para promover una experiencia subjetiva satisfactoria, estimulando el desarrollo de sus competencias socioemocionales. La invitación, por tanto, es a mirar la trayectoria real de cada estudiante desde su singularidad, respetando y valorando las diferencias; constituyendo un recorrido único para cada estudiante, a lo largo de los diversos años, ciclos y niveles de la experiencia escolar, en el cual desarrolla un proceso de construcción individual de un proyecto vital de sentido, como persona y ciudadano.

Finalmente, frente al riesgo de fracaso escolar, las recomendaciones sugeridas por la literatura se focalizaron principalmente en la intervención temprana, en la mejora de la experiencia escolar y en una estrategia personalizada con los estudiantes en sus centros educativos. En este sentido, el compromiso escolar, como herramienta conceptual y dispositivo de medición, puede ser considerado una estrategia de monitoreo del proceso de aprendizaje y de recogida de información. Lo anterior posibilitaría contar con insumos válidos y confiables, tanto para los organismos gubernamentales, como también, para el acompañamiento a los centros educativos en la promoción de trayectorias educativas plenas.

\section{Financiamiento}

Este artículo ha recibido el apoyo del proyecto FONDEF ID14I20078 "Medición del compromiso del niño, niña y adolescente con sus estudios para la promoción de trayectorias educativas exitosas" financiado por la Comisión Nacional de Investigación Científica y Tecnológica CONICYT, Chile.

\section{Referencias}

Acevedo, F. (2015). Una aproximación al fenómeno de desafiliación educativa en la transición hacia la Educación Superior en el interior de Uruguay. Páginas de Educación, 7(1), 131-148. doi:10.22235/pe.v7i1.588

Acevedo, F., \& Menni, G. (2016). Programas de inclusión educativa implementados en la Educación Media pública de Uruguay: Breve estado del arte y una mirada hacia el programa Compromiso Educativo. En Dalla Corte y Carneiro Sarturi (Eds.), Políticas Públicas da Educação Básica e Superior. Santa Maria: Universidad Federal de Santa Maria (RS, Brasil).

Acevedo, F. (2020). Factores explicativos del abandono de los estudios en la educación superior en contextos socio-académicos desfavorables. Revista Española de Pedagogía, 78(276), 253-269.

doi:10.22550/REP78-2-2020-02

Agencia de Calidad de la Educación de Chile. (2019a). Resultados Educativos 2019. Santiago, Chile: Gobierno de Chile. Recuperado de http://www.inee.edu.mx/bie_wr/mapa_indica/ 
2016/PanoramaEducativoDeMexico/RE/RE00 /2016_RE00__Intro.pdf

Agencia de Calidad de la Educación de Chile. (2019b). PISA 2018 Entrega de Resultados. Competencia Lectora, Matemática y Científica en estudiantes de 15 años en Chile. Santiago, Chile: Gobierno de Chile. Recuperado de: http://archivos.agenciaeducacion.cl/PISA_201 8-Entrega_de_Resultados_Chile.pdf

Arteaga, I., \& Glewwe, P. (2019). Do community factors matter? An analysis of the achievement gap between indigenous and non-indigenous children in Peru. International Journal of Educational Development, 65, 8091. doi:10.1016/j.ijedudev.2017.08.003

Baeza, A., \& Lamadrid, S. (2018). Trayectorias educativas según género. Lo invisible para la política educativa chilena. Revista de Investigación Educativa, 36(2), 471-490. doi:10.6018/rie.36.2.298061

Bayón-Calvo, S., Corrales-Herrero, H., \& De Witte, K. (2020). Assessing regional performance against early school leaving in Spain. International Journal of Educational Research, 99. doi:10.1016/j.ijer.2019.101515

Bayon-Calvo, S. (2019). An analysis of early school leaving in Spain: Some keys for the educational policy at the dawn of the $21 \mathrm{st}$ century. Revista Complutense de Educación, 30(1), 35-53. doi: 10.5209/RCED.55465

Benavente, M., Cova, F., Pérez-Salas, C., Varela, J., Alfaro, J., \& Chuecas, J. (2017). Propiedades psicométricas de la Escala Breve de Bienestar Subjetivo en la Escuela para Adolescentes (BASWBSS) en una muestra de adolescentes Chilenos. Revista Iberoamericana de Diagnóstico y Evaluación - Avaliação Psicológica, 48(3), 53-65. doi:10.21865/RIDEP48.3.05

Bermejo-Paredes, S., \& Maquera-Maquera, Y. A. (2019). Interpretation of the andean rural school in aimara communities of Puno-Perú. Revista Electrónica Educare, 23(2), 1-15. doi:10.15359/ree.23-2.4

Bonilla, C., \& Tejada I. (2016). Unanswered questions in Colombia's foreign language education policy. Profile, Issues in Teachers' Professional Development, 18(1), 185-201. doi:10.15446/profile.v18n1.51996
Bourdieu, P., \& Passeron, J.-C. (1979). La reproducción. Elementos para una teoría de la enseñanza. Barcelona: Laia S.A.

Bravo-Sanzana, M., Pavez, M., Salvo-Garrido, S., \& Mieres-Chacaltana, M. (2019). Autoeficacia, expectativas y violencia escolar como mediadores del aprendizaje en Matemática. Revista Espacios, 40(33), 28.

Bravo-Sanzana, M., Salvo, S., Mieres-Chacaltana, M., Mansilla, J., \& Hederich, C. (2017). Perfiles de desempeño académico: la importancia de las expectativas familiares. Perfiles Latinoamericanos, 25(50), 361-386. doi:10.18504/pl2550-016-2017

Bravo-Sanzana, M., Salvo Garrido, S., \& Muñoz Poblete, C. (2015). Profiles of Chilean students according to academic performance in mathematics: An exploratory study using classification trees and random forests. Studies in Educational Evaluation, 44, 50-59. doi:10.1016/j.stueduc.2015.01.002

Cabrera, L. (2019). Políticas educativas preventivas de la repetición de curso en la enseñanza obligatoria en España. Multidisciplinary Journal of Educational Research, 9(3), 227-257. doi:10.17583/remie.2019.4523

Cardenas, T (1998). Justicia Social y Educación: por los caminos de la esperanza y la desigualdad en México. En F. Knight. XXI International Congress of the Latin American Studies Association. The Latin American Studies Association (LASA). Chicago: Illinois.

Castellar, E. (2015). Política de gratuidad: un estudio de caso. Zona Próxima, 23(2), 1-15.

Caro, L., \& Flores, N. (2018). Programas educativos con uso de TIC en la región Bogotá Cundinamarca - Colombia- un modelo de evaluación. EDMETIC, 7(1), 297320. doi:10.21071/edmetic.v7i1.6746

Casarrubia-Ruíz, J., \& Ramos, D. (2018) Políticas de formación docente en Colombia (19942014). El caso de Montería. Panorama, 12(23), 31-50. doi:10.15765/pnrm.v12i23.1199

Charry, P. (2019). Hacía una educación rural inclusiva. Revista Nacional e Internacional de Educación Inclusiva, 12(1), 225-246.

Contreras, D., Elacqua, G., Martinez, M., \& Miranda, Á. (2016). Bullying, identity and 
school performance: Evidence from Chile. International Journal of Educational Development, 51, 147-162. doi:10.1016/j.ijedudev.2016.09.004

Cortázar, A., Molina, M., Sélman, J., \& Manosalva, A. (2020). Early childhood education effects on school outcomes: Academic achievement, grade retention and school drop Out. Early Education and Development, 31(3), 376-394, doi:10.1080/10409289.2019.1666445

Cox, C., \& Meckes, L. (2016). International largescale assessment studies and educational policy-making in Chile: Contexts and dimensions of influence. Reserach Papers in Education, 31(5), 502-515. doi:10.1080/02671522.2016.1225349

D’alessandre, V. (2014). El desafío de universalizar el nivel medio. Trayectorias escolares y curso de vida de los adolescentes y jóvenes latinoamericanos. IIPE - UNESCO Sede Regional Buenos Aires. Organización de Estados Iberoamericanos Para la Educación, la Ciencia y la Cultura (OEI).

De Armas, G. (2017). Trayectorias educativas en Uruguay. Principales rasgos, tendencias y desafíos para las políticas públicas. Montevideo: UNICEF.

De Melo, G., \& Machado, A. (2016). Trayectorias educativas. Evidencia para Uruguay. Documento de trabajo. Montevideo: Instituto Nacional de Evaluación Educativa.

De la Vega Rodríguez, L., \& Picazo Verdejo, M. (2016). La accountability de la calidad y equidad de una política educativa: El caso de la subvención escolar preferencial para la infancia vulnerable en Chile. Revista del CLAD Reforma y Democracia, (65),193-224.

De Miguel-Luken, V., \& Solana-Solana, M. (2016). Immigrants in the Educational System in Spain: Who Persists?. Social Indicators Research, 132, 733-755. doi:10.1007/s11205-016-1323-4

Espinoza, O., González, L., McGinn, N., \& Castillo, D. (2020). Engaging dropouts with differentiated practices: Some evidence from Chile. Research Papers in Education. doi:10.1080/02671522.2020.1736615

Espinoza, O., González, L., McGinn, N., \& Castillo, D. (2019). What factors predict the engagement of dropouts in alternative secondary schools in Chile? Improving Schools, 23(1), 47-67. doi:10.1177/1365480219864835

Espinoza, O. (2015). La Investigación en/sobre Políticas Educacionales en Chile: Panoramas y Perspectivas. EccoS Revista Científica, (37), 233-257. doi:10.5585/EccoS.n37.5692

Essomba Gelabert, M. A., Guardiola Salinas, J., \& Pozos Perez, K. V. (2019). Alumnado de origen extranjero y equidad educativa. Propuestas para una política educativa intercultural en España hoy. Revista Interuniversitaria de Formación Del Profesorado, 33(2), 43-61. doi:10.9685/73219

Fernández-Mellizo, M., \& Martínez-GarcíaI, J. (2017). Inequality of educational opportunities: School failure trends in Spain (1977-2012). International Studies in Sociology of Education, 26(3), 267-287. doi:10.1080/09620214.2016.1192954

Ferreiro, F. J., Ríos, D., \& Álvarez, D. (2016). Influencia del entorno familiar en el rendimiento académico en Galicia (España). Revista Iberoamericana de Educación, 70(1), 47-62.

Flotts, M. P., Manzi, J., Jiménez, D., Abarzúa, A., Cayuman, C., \& García, M. J. (2016). Informe de Resultados TERCE, Logros de Aprendizaje. Laboratorio Latinoamericano de Evaluación de la Calidad de la Educación. Orealc/Unesco Santiago.

Gálvez-Nieto, J., Vera-Bachman, D., Trizano, I., \& García, J. (2015). Examen psicométrico de la Escala de Actitudes hacia la Autoridad Institucional (AAI-A), en Estudiantes Chilenos. Revista Iberoamericana de Diagnóstico y Evaluación - e Avaliação Psicológica, 1(39),57-67.

García, P. (2018). Políticas educativas para el apoyo de trayectorias escolares en el nivel medio en América Latina: La provisión de recursos como estrategia. Revista Fuentes, 20(2), 37-50. doi:10.12795/revistafuentes.2018.v20.i2.03

Gazmuri, C., Manzi, J., \& Paredes, R. (2015). Disciplina, clima y desempeño escolar en Chile. Revista CEPAL, 115. Recuperado de: https://repositorio.cepal.org/bitstream/handle/ 11362/37833/1/REV115ManziParedes_es.pdf 
Gimenez-Martínez, M. A. (2015). Great projects and crude realities Advances and retreats of the educational policy in contemporary Spain. History of Education Review, 44(2), 186-202. doi:10.1108/HER-11-2013-0020

Guarnizo, M., Velásquez, J., Jiménez, P., \& Alzate, B. (2019). Educación para el emprendimiento: Análisis y aportes a la política pública de educación en Colombia desde la sistematización de experiencias en instituciones de educación básica y media. Revista Complutense de Educación, 30(1), 225-243. doi:10.5209/RCED.57165

Guevara, R., \& Téllez, S. (2019). From the Indicative to the Imperative. Colombia, the most educated in 2025? En C. Ornelas (ed.), Politics of Education in Latin America. Boston: Brill Sense.

Guio, J., Choi, Á., \& Escardíbul, J.O. (2018). Labor markets, academic performance and school dropout risk: evidence for Spain, International Journal of Manpower, 39(2), 301-318. doi:10.1108/IJM-08-2016-0158

Janosz, M., Archambault, I., Morizot, J., \& Pagani, L. (2008). School engagement trajectories and their differential predictive relations to dropout. Journal of Social Issues, 61(1), 21-40. doi:10.1111/j.1540-4560.2008.00546.x

Lara, L., Saracostti, M., Navarro, J.-J., de-Toro, X., Miranda-Zapata, E., Trigger, J. M., \& Fuster, J. (2018). Compromiso escolar: Desarrollo y validación de un instrumento. Revista Mexicana de Psicología, 35(1), 52-62.

Latorre, M. (2018). Fracaso escolar en estudiantes de educación secundaria de colegios públicos de Perú, 2005-2010, y sus factores asociados. Revista EDUCA UMCH, 11, 13-50. doi:10.35756/educaumch.v11i0.67

León, G., \& Valdivia, M. (2015). Inequality in school resources and academic achievement: Evidence from Peru. International Journal of Educational Development, 40, 71-84. doi:10.1016/j.ijedudev.2014.11.015

Ley $\mathrm{N}^{\circ} 28044,29$ de junio de 2003, Ley General de Educación. núm. 8437, pp. 248943 248956. Diario El Peruano, 29 de junio de 2003.

López, E., \& González, A. L. (2018). External Evaluation Tests as Focal Points of Educational Policy and its Location in Spain.
HSE-Social and Education History, 7(3), 232255. doi:10.17583/hse.2018.2836

López, V., Oyanedel, J. C., Bilbao, M., Torres, J., Oyarzún, D., Morales, M., Ascorra, P., \& Carrasco, C. (2017). School achievement and performance in Chilean high schools: The mediating role of subjective wellbeing in school-related evaluations. Frontiers in Psychology, 8, 1189. doi:10.3389/fpsyg.2017.01189

Martínez-Novillo, J. (2017a). Dispositivos institucionales y gestión del fracaso escolar: Las paradojas de la atención a la diversidad en la ESO. Cuadernos de Relaciones Laborales, 35(2), 327-345. doi: 10.5209/CRLA.56776

Martínez-Novillo, J. (2017b). La construcción del «fracaso escolar» en España. Génesis y cristalización de un problema social. Revista de Sociología, 102(3), 477-507. doi:10.5565/rev/papers.2297

Decreto 2370, Ministerio de Educación Nacional de Colombia (2019).

Miranda-Zapata, E., Lara, L., Navarro, J. J., Saracostti, M., \& de-Toro, X. (2018). Modelling the effect of school engagement on attendance to classes and school performance. Revista de Psicodidáctica (English ed.), 23(2), 102-109. doi:10.1016/j.psicod.2018.02.003

Monarca, H. (2017). Políticas públicas de apoyo educativo y configuración de trayectorias escolares. Magis, Revista Internacional De Investigación En Educación, 10(20), 69-84. doi:10.11144/Javeriana.m10-20.ppae

Montecinos, C., Castro, G., Díaz, R., Manríquez, L., \& Edwards, A. (2018). Trabajo interprofesional en los Centros Escolares: Cambiando trayectorias de vulnerabilidad a la deserción en estudiantes secundarios. Revista Electrónica de Investigación y Evaluación Educativa. 24(2). doi:10.7203/relieve.24.2.13386

Morentin-Encina, J., Ballesteros, B., \& Mateus, S. (2019). ¿Igualdad de oportunidades? Más que el mero acceso: Narrativas de jóvenes con trayectorias de fracaso y abandono temprano de la educación en España y Portugal. Revista Fuentes, 21(2), 143-159. doi:10.12795/revistafuentes.2019.v21.i2.01

Muelle, L. (2018). Factores socioeconómicos y contextuales asociados al bajo rendimiento 
académico de alumnos peruanos en PISA 2015. Apuntes. Revista de Ciencias Sociales, 47(86). doi:10.21678/apuntes.86.943

Murillo, F. J., \& Garrido, C. M. (2017). Segregación Social en las escuelas públicas y privadas en América Latina. Educação \& Sociedade, 38(140), 727-750. doi:10.1590/es0101-73302017167714

Naranjo, D. \& Carrero, A. (2017) Retos y desafíos de la Educación rural para niños y jóvenes en escenarios de Construcción de Paz: Una mirada desde lo local para la transformación global. Prospectiva: Revista de Trabajo Social e intervención social, 24, 95-120.

OECD. (2018). España - Nota del país Resultados de PISA 2018. OECD. Recuperado de:

https://www.oecd.org/pisa/publications/PISA2 018_CN_esp_ESP.pdf

ONU. (1948). Declaración Universal De Derechos Humanos. Organización de las Naciones Unidas. París: ONU. Recuperado de:

https://www.ohchr.org/EN/UDHR/Documents /UDHR_Translations/spn.pdf

Ortiz, L., \& Betancourt, C. (2020). Evaluación del Programa de Aceleración del Aprendizaje: Una apreciación estratégica hacia la educación inclusiva en el posconflicto. Praxis y Saber, 11(25), 97-110. doi:10.19053/22160159.v11.n25.2020.8207

Pàmies Rovira, J., \& Bertran Tarrés, M. (2018). Factors afecting educational success and continuity among Young people of Moroccan descent in Catalonia (Spain). IJERI: International Journal of Educational Research and Innovation, (10), 179-189.

Pérez, F. (2018). Políticas educativas en Colombia: En busca de la calidad. Actualidades Pedagógicas, 71, 193-213. doi:10.19052/ap.4430

Rivas, A., \& Sanchez, B. (2020). Race to the classroom: the governance turn in Latin American education. The emerging era of accountability, control and prescribed curriculum, Compare: A Journal of Comparative and International Education, Latest Articles. doi:10.1080/03057925.2020.1756745
Román, M. (2013). Factores asociados al abandono y la deserción escolar en América Latina: una mirada en conjunto. REICE. Revista Iberoamericana sobre Calidad, Eficacia y Cambio en Educación, 11(2), 3359.

Rumberger, R. W., \& Rotermund, S. (2012). The relationship between engagement and high school dropout. En S. L. Christenson, A. L. Reschly, \& C. Wylie (Eds.), Handbook of research on student engagement. (pp. 491513). Springer Science + Business Media.

Saracostti, M., Lara, L., \& Miranda-Zapata, E. (2019). Plataforma tecnológica de evaluación del compromiso escolar, factores de contexto y reporte de resultados online: Breve Reporte Técnico, Electronic Journal of Research in Educational Psychology, 17(1), 193-212. doi:10.25115/ejrep.v17i47.2049

Sillard, M., Garay, M., \& Troncoso, I. (2018). Análisis al nuevo sistema de admisión escolar en Chile: la Región de Magallanes como experiencia piloto. Revista Calidad En La Educación, 49, 112-136. doi:10.31619/caledu.n49.578

Simões, C., Rivera, F., Moreno, C., \& Matos, M. (2018). School performance paths: Personal and contextual factors related to top performers and low achievers in Portugal and Spain. The Spanish Journal of Psychology, 21, E36. doi:10.1017/sjp.2018.37

SITEAL. (2019). Inclusión y equidad educativa. Buenos Aires, Argentina: Instituto Internacional de Planeamiento de la Educación UNESCO. Recuperado de: https://www.siteal.iiep.unesco.org/sites/defaul t/files/sit_informe_pdfs/siteal_inclusion_y_eq uidad_20190525.pdf

Silva, M. (2016). Competencias de estudiantes iberoamericanos al finalizar la educación secundaria alta. Perfiles educativos, 38(152), 88-108.

Tarabini, A., Curran, M., Montes, A., \& Parcerisa, L. (2016). The politics of educational success: A realist evaluation of early school leaving policies in Catalonia (Spain). Critical Studies in Education, 59(3), 364-381. doi:10.1080/17508487.2016.1197842

Terigi, F. (2009). El fracaso escolar desde la perspectiva psicoeducativa: Hacia una 
reconceptualización situacional. Revista Iberoamericana de Educación, 50, 23-39.

Terigi, F. (2014). Trayectorias escolares $e$ inclusión educativa: Del enfoque individual al desafio para las políticas educativas, en Marchesi, A.; Blanco, R. y Hernández, L. (coordinadores), Avances y desafíos de la educación inclusiva en Iberoamérica. Metas Educativas 2012. Madrid: Organización de Estados Iberoamericanos para la Educación, la Ciencia y la Cultura (OEI). http://www.bnm.me.gov.ar/giga1/documentos /EL004307.pdf

Toscano, A. G., Briscioli, B., \& Morrone, A. (2015). Trayectorias escolares: estrategias teórico-metodológicas para su abordaje. XI Jornadas de Sociología. Facultad de Ciencias Sociales, Universidad de Buenos Aires, Buenos Aires.

Tovar, M., \& Ríos, C. (2017). Trabajo infantil y deserción escolar en el Perú al 2015. Apuntes de Ciencia \& Sociedad, 7(1). doi:10.18259/acs.2017008

UNESCO. (2012). La pérdida de oportunidades: el impacto de la repetición y el abandono escolar. Compendio Mundial de la Educación de la UNESCO (2012). Recuperado de http://www.uis.unesco.org/Education/GED\%2 0Documents\%20C/ged-2012-sp.pdf

Venegas-Muggli, J. (2020). Secondary School Context and (Timely) College completion in Chile's education system: A cohort study. Journal of College Student Retention: Research, Theory \& Practice. doi:10.1177/1521025120910477

Virtanen, M., Kivimaki, M., Luopa, P., Vahtera, J., Elovainio, M., Jokela, J., \& Pietikainen, M. (2009). Staff reports of psychosocial climate at school and adoles-cents' health, truancy and health education in Finland. European Journal of Public Health, 19(5), 554-560. doi:10.1093/eurpub/ckp032

Weinstein, J., \& Villalobos, C. (2016). Chile: La experiencia de la Subvención Escolar Preferencial. En M. Souto (Coord.) Transferencias directas a escuelas: reflexiones sobre prácticas en América Latina (pp. 58100). Buenos Aires: IIPE-UNESCO. 